\title{
Predictores de mortalidad en pacientes con sepsis obstétrica mediante uso de score de sepsis obstétrica y evaluación secuencial de falla orgánica-obstétrica
}

\author{
Blanca E Herrera Morales* Juan Lara Cruz, ${ }^{*}$ Victoria Ortega López ${ }^{\star}$
}

\section{RESUMEN}

Objetivo: Determinar los predictores de mortalidad en pacientes con sepsis obstétrica mediante score de sepsis obstétrica (SOS) y evaluación secuencial de falla orgánica-obstétrica (SOFA-O).

Material y métodos: Se realizó un estudio observacional, retrospectivo, descriptivo, donde se recabaron los datos de pacientes que ingresaron a una Unidad de Cuidados Intensivos de hospitales de segundo nivel, en el periodo 30 de junio de 2015 al 30 de junio de 2017 y que tuvieron diagnóstico de sepsis obstétrica, donde se aplicaron instrumentos mediante escala SOS y SOFA-O, correlacionándose las variables con mortalidad materna.

Resultados: De un universo de 284 pacientes que ingresaron a $\mathrm{UCI}$ de Hospitales de segundo nivel se seleccionaron 51 , quienes tenían criterios de inclusión para sepsis, correlacionándose con variables de escala de SOS y SOFA-O, encontrando como mayor factor de riesgo para el desarrollo de sepsis ser multigesta, tener preeclampsia, anemia, cesárea, mal control prenatal y tener procedimientos invasivos. Las variables cuantitativas relacionadas con muerte materna fueron creatinina, relación $\mathrm{PaO}_{2} / \mathrm{FiO}_{2}$, frecuencia cardiaca, lactato, saturación venosa, con un puntaje de SOS mínimo y máximo para muerte materna (7-22) y para SOFA-O (10-18) puntos. Hubo una mortalidad de $7.8 \%$ (cuatro pacientes) de la población estudiada. Conclusiones: La incidencia de sepsis obstétrica se encuentra en aumento, por lo que el reconocimiento rápido de ésta y la terapia adecuada impactarán en la supervivencia de la paciente. Palabras clave: Escala SOS, SOFA-O, sepsis obstétrica, mortalidad.

\section{SUMMARY}

Objective: To determine the predictors of mortality in patients with obstetric sepsis using Sepsis Obsessional Score (SOS) and Sequential Organ Failure Assessment-Obstetric (SOFA-O).

Material and methods: An observational, retrospective, descriptive study was carried out, where data were collected from patients who entered an intensive care unit of second level hospitals in the period June 30 2015, to June 302017 who had a diagnosis of sepsis Obstetric, where SOS and SOFA-O instruments were applied, correlating the variables with maternal mortality.

Results: From a universe of 284 patients who entered the ICU of second level hospitals, 51 patients were selected who had inclusion criteria for sepsis, correlating with SOS and SOFA-O scale variables, finding it to be a major risk factor for development of sepsis being multigested, having preeclampsia, anemia, cesarean section, prenatal poor control and having invasive procedures, the quantitative variables related to maternal death were: creatinine, $\mathrm{PaO}_{2} / \mathrm{FiO}_{2}$ ratio, heart rate, lactate, venous saturation. With a minimum and maximum SOS score for maternal death (7-22) and SOFA-O (10-18) points. There was a mortality of $7.8 \%$ (four patients) of the study population.

Conclusions: The incidence of obstetric sepsis is increasing, so rapid recognition and appropriate therapy will impact patient survival.

Key words: Scale SOS, SOFA-O, obstetric sepsis, mortality.

\section{RESUMO}

Objetivo: Determinar os preditores de mortalidade em pacientes com sepse obstétrica pelo Escore de Sepse Obstétrica (S.O.S.) e Avaliação Seqüencial de Falha Orgânico-Obstétrica (SOFA-O).

Material e métodos: Foi realizado um estudo observacional, retrospectivo, descritivo, onde foram coletados dados de pacientes internados em uma unidade de terapia intensiva no período de 30 de junho de 2015 a 30 de junho de 2017 com diagnóstico de sepse obstétrica, onde foram aplicados instrumentos utilizando escalas S.O.S e SOFA-O, correlacionando as variáveis com a mortalidade materna. Resultados: De um universo de 284 pacientes internados na UTI, foram selecionados 51 pacientes que possuíam critérios de inclusão para sepse, correlacionando com as variáveis S.O.S e SOFA-O, encontrando como um importante fator de risco para o desenvolvimento da sepse ser multigesta, apresentar pré-eclâmpsia, anemia, cesárea, controle pré-natal deficiente e

\footnotetext{
* Departamento de Terapia Intensiva. Hospital General Regional Núm. 196 IMSS Ecatepec.

Recepción: 01/09/2017. Aceptación: 06/09/2018.

Este artículo puede ser consultado en versión completa en http://www.medigraphic.com/medicinacritica
}

procedimentos invasivos. As variáveis quantitativas relacionadas ao óbito materno foram creatinina, relação $\mathrm{PaO}_{2} / \mathrm{FiO}_{2}$, frequência cardíaca, lactato, saturação venosa. Com um escore de S.O.S mínimo e máximo para morte materna (7-22) e para SOFA-O (10-18). Houve uma mortalidade de $7.8 \%$ (4 pacientes) da população estudada.

Conclusões: A incidência de sepse obstétrica está aumentando, portanto o reconhecimento rápido e a terapia adequada terão impacto na sobrevida do paciente.

Palavras-chave: Escore S.O.S, SOFA-O, sepse obstétrica, mortalidade.

\section{INTRODUCCIÓN}

La infección puerperal es una de las principales causas de morbimortalidad prevenibles de la paciente obstétrica. La sepsis materna es la principal causa de muerte materna y representa $15 \%$ de las muertes maternas en todo el mundo. ${ }^{1}$ En México, durante la primera semana de abril del 2017 se registraron 155 defunciones, siendo en 11 pacientes sepsis obstétrica con un porcentaje de $6 \%$, lo cual la pone en sexto lugar de todas las causas de muerte materna. ${ }^{2,3}$ Se espera que las incidencias de las infecciones puerperales continúen aumentando en el futuro, como resultado del cambio demográfico de la población de mujeres embarazadas, del incremento sostenido de las tasas de nacimiento por cesárea, de los embarazos en mujeres mayores de 40 años, por la epidemia mundial de la obesidad y diabetes mellitus tipo 2, nuevos procedimientos terapéuticos intrauterinos y la resistencia bacteriana a los antibióticos. ${ }^{4}$ Los datos publicados por Vásquez, Estenssoro y cols. en 2007, se refieren a la sepsis de origen pélvico como la segunda causa, responsable del $16 \%$ del ingreso obstétrico a la Unidad de Cuidados Intensivos. La incidencia de sepsis puerperal varía por condiciones socioeconómicas, como la pobreza y la falta de accesibilidad a control prenatal y puerperal, por centros que no cuentan con condiciones adecuadas y esenciales o con profesionales capacitados para asistir partos o cesáreas ni para el manejo oportuno de las complicaciones. ${ }^{5}$

EI CIE 10 define sepsis obstétrica como un aumento de la temperatura por encima de $38.0^{\circ} \mathrm{C}$ que se mantiene durante más de 24 horas o recurrente entre el final del primer y décimo día después del parto, aborto involuntario, o la terminación del embarazo. La OMS define sepsis puerperal como la infección del tracto genital que ocurre en cualquier momento entre el comienzo de la ruptura de membranas o trabajo de parto y los 42 días postparto, con la presencia de fiebre y uno o más de los siguientes síntomas: dolor pélvico, loquios o flujo vaginal anormal, olor anormal y retraso en la velocidad 
de involución del útero. ${ }^{5-7}$ Los organismos que causan infección son, por lo general, de la flora polimicrobiana del tracto genitourinario. Las infecciones obstétricas comunes se ven agravadas por las alteraciones fisiológicas del embarazo y tienen el potencial de progresar a choque séptico. ${ }^{4,8,9}$ Las causas de la sepsis puerperal pueden ser resultado de condiciones obstétricas y no obstétricas; entre ellas se encuentran, causas obstétricas: corioamnionitis, endometritis, aborto séptico, infección de la herida, episiotomía y desgarro vaginal. Otras causas relacionadas son: infección del tracto urinario inferior, pielonefritis, infección de mama absceso y/o mastitis, tromboflebitis séptica pélvica, vía de nacimiento (vaginal o cesárea) y ruptura prematura de membranas. Dentro de las causas no obstétricas: virus de inmunodeficiencia humana, neumonía, tuberculosis y malaria. ${ }^{8}$ Entre los factores de riesgo asociados se encuentran: número de exámenes vaginales. Obesidad, ambiente quirúrgico y salas de partos que no cuentan con normativas de seguridad del paciente, hemorragia intraparto o postparto (pérdida hemática mayor de 500 $\mathrm{mL})$. Anemia-malnutrición-desnutrición, trastornos inmunitarios, diabetes mellitus materna, bajo nivel socioeconómico y pobre control prenatal. . $^{3,9,10-12}$

Dentro de los agentes microbiológicos, se trata generalmente de infecciones polimicrobianas (gérmenes de la flora del tracto genital). Los gérmenes aislados con mayor frecuencia son: Aerobios: estreptococos (A, B), enterococos. Escherichia coli, Klebsiella y Staphylococcus aureus. Anaerobios: Bacteroides, especies de Peptostreptococcus, Clostridium y Fusobacterium. Otros: Ureaplasma Urealyticum, Mycoplasma h. y Chlamydia trachomatis. ${ }^{10-12}$ El efecto sinérgico de la combinación bacteriana y de factores de riesgo, así como la presencia de tejido desvitalizado o condiciones generales de resistencia disminuida en el huésped, incrementa exponencialmente la virulencia y patogenicidad de estos gérmenes, generando condiciones propicias para una sepsis puerperal. ${ }^{12}$

El diagnóstico se basa en: Anamnesis: antecedentes, procedencia (sala de partos o internación, quirófano, domicilio, etc.), signos, síntomas, momento de inicio, factores de riesgo, comorbilidades, etc. Examen físico obstétrico y general: enfocado a la identificación de la fuente de infección. Estudios de laboratorio en sangre y orina, estudios de bacteriología como hemocultivos, urocultivos, material de colecciones o de heridas complicadas. La toma del material se debe realizar antes de instaurar el tratamiento antibiótico. Diagnóstico por imágenes: radiografía de tórax, abdomen, ecografía pélvica, abdominal o de partes blandas, valorando tomografía computarizada o resonancia magnética. El diagnóstico y tratamiento intensivo inicial en el lapso de 1-6 horas disminuye la mortalidad por sepsis de 37.2 a $30.8 \% .^{9}$
La evaluación sistémica orienta a la localización del foco séptico; se pueden observar: fiebre, escalofríos, hipotensión arterial, náuseas, vómito, diarrea, íleo, herida quirúrgica con eritema, induración, dolor y supuración, dolor abdominal o pelviano, reacción peritoneal, útero subinvolucionado, loquios fétidos, alteración del sensorio, eritema (rash) cutáneo maculopapular, mamas eritematosas e induradas, con sintomatología urinaria, respiratoria, entre otras. ${ }^{4,13}$ Los indicadores de mal pronóstico en choque séptico en obstetricia son el retraso en el diagnóstico inicial, mala respuesta a la reanimación masiva con fluidos intravenosos, gasto cardiaco bajo, reducción de la extracción de oxígeno, lactato sérico elevado (mayor de $4 \mathrm{~mol} / \mathrm{L}$ ) y síndrome de falla multiorgánica. ${ }^{14}$

El tratamiento está enfocado a evitar falla orgánica; las primeras medidas terapéuticas deben realizarse en las salas o servicios de obstetricia, sin esperar el traslado de la paciente a UCl. ${ }^{14}$ Una vez que ingresa la puérpera a $\mathrm{UCl}$, el manejo no difiere del habitual en casos de sepsis y choque séptico. El tratamiento médico antibiótico debe preceder y acompañar al tratamiento quirúrgico. En general, se trata de infecciones polimicrobianas: se recomienda un esquema antibiótico intravenoso combinado, sin demoras ante la sospecha del cuadro, dentro de la primera a la sexta hora, al mismo tiempo que la reposición de volumen («la hora de oro»). .,14 Los esquemas antibióticos intravenosos combinados que se recomiendan son: ceftriaxona + clindamicina + ampicilina. Betalactámicos + aminoglucósidos. Clindamicina + aminoglucósidos o quinolonas. Betalactámicos + aminoglucósidos + metronidazol. Cefalosporinas de tercera generación + quinolona. Carbapenémicos + vancomicina. ${ }^{14}$

Se deberá mantener volemia adecuada según necesidad y guías de práctica clínica o protocolos del servicio; sin embargo, en el puerperio la posibilidad de edema pulmonar por sobrecarga de volumen es mayor, por lo que se debe establecer un manejo cauteloso del balance de ingresos y egresos y presión venosa central. ${ }^{4,13}$ Para sostén hemodinámico y para lograr tener una PAM mayor de $65 \mathrm{mmHg}$ y diuresis mayor de $0.5 \mathrm{~mL} / \mathrm{kg} / \mathrm{h}$ : dopamina, noradrenalina o dobutamina, según sea el caso. En cuanto a glucemia, se debe mantener idealmente entre 80-110 mg/dL, sin superar los $150 \mathrm{mg} / \mathrm{dL}$, evaluando la necesidad de administración de insulina intravenosa, si se observa tendencia a la hiperglucemia. El abordaje quirúrgico es uno de los pilares fundamentales en el tratamiento de la sepsis obstétrica y amerita una toma de decisión consensuada entre obstetra, intensivista o anestesiólogo. La demora de esta parte del tratamiento lleva a la paciente a un punto de irreversibilidad clínica. Debe considerarse la posibilidad de la histerectomía si hay formación de abscesos, gangrena gaseosa, necrosis séptica de la histerorrafia, o si se deterioran las condicio- 
nes maternas, sin respuesta, a pesar de una terapéutica apropiada, con persistencia de sensibilidad uterina, loquios fétidos, fiebre o choque refractario al tratamiento. La campaña sobreviviendo a la sepsis recomienda el uso de corticoesteroides intravenosos (hidrocortisona 200$300 \mathrm{mg} / \mathrm{dL}$ durante siete días en 3-4 dosis divididas o por infusión continua) en los pacientes con shock séptico que requieran vasopresores. ${ }^{11}$ Otras estrategias terapéuticas son la tromboprofilaxis con heparinas de bajo peso molecular, protección gástrica de úlceras por estrés y nutrición enteral temprana. $4,10,13$

\section{Escala SOFA-O para sepsis obstétrica}

En la puntuación SOFA, disfunción cardiovascular se basa en la disfunción y requerimientos de vasopresores. La escala de morbimortalidad sigue siendo escasa. A pesar de que la puntuación SOFA muestra un buen valor pronóstico en pacientes obstétricas, no debe considerarse definitiva. El parámetro fisiológico más importante que parece ajustarse es $\mathrm{PaO}_{2} / \mathrm{FiO}_{2}$ de acuerdo a la fisiología respiratoria de una paciente embarazada, por lo que esta escala posee dos cambios: primero, el valor inferior de $\mathrm{PaO}_{2} / \mathrm{FiO}_{2}$ en la columna de 3 puntos, basada en la disminución de la capacidad pulmonar en el embarazo y ser la media-1SD para Kirby; segundo, agregando el archivo de tiempo con sepsis. Después de estas correcciones, se puede determinar la sensibilidad y la especificidad para la predicción de muerte materna. En un estudio realizado en 2016, Blanco Esquivel y colaboradores realizaron comparación con la escala SOFA versus SOFA-O donde encontraron área mayor bajo la curva (AUC) para O-SOFA $(0,868$, $p<0.001)$ que SOFA $(0.796, p=0.003) .{ }^{11,12}$

\section{Escala para el reconocimiento de sepsis en obstetricia SOS (Sepsis Obstetrics Scoring System)}

La escala de sepsis en Obstetricia fue creada mediante la modificación de los sistemas de puntuación validados de acuerdo con los cambios fisiológicos del embarazo reconocidos. Esta es la principal razón a la que se debe la sobrestimación de las otras escalas al no tener en cuenta estos cambios, los cuales incluyen disminución de la presión diastólica en 5-10 $\mathrm{mmHg}$ en el segundo trimestre, con retorno en el tercer trimestre, aumento en el promedio de la frecuencia cardiaca en 17\%, elevación en el recuento leucocitario en 16,900 para el tercer trimestre y 30,000 en el trabajo de parto.

Cabe resaltar que, en parámetros como temperatura, frecuencia respiratoria, presión sistólica, porcentaje de saturación de oxígeno y porcentaje de formas inmaduras de línea blanca, no se producen cambios respecto a la población en general. El puntaje SOS tiene como objetivo identificar el riesgo de ingreso a $\mathrm{UCl}$ de una paciente gestante o puérpera con signos de sepsis, teniendo como punto de corte una puntuación mayor o igual a 6 . Se documentó una frecuencia de $16.7 \%$ de pacientes con este puntaje entre todas las 850 pacientes que se incluyeron en este estudio y se obtuvo una sensibilidad de $88.9 \%$, especificidad de $95.2 \%$, valor de predicción positivo (VPP) de 16.7\% y negativo (VPN) de $99.9 \%$. $^{10,11}$

\section{MATERIAL Y MÉTODOS}

Se realizó un estudio observacional, retrospectivo, descriptivo, donde se recabaron los datos de pacientes quienes ingresaron a una Unidad de Cuidados Intensivos de hospitales de segundo nivel, en el periodo 30 de junio 2015 al 30 de junio 2017 y que tuvieron diagnóstico de sepsis obstétrica, donde se aplicaron instrumentos mediante escala SOS y SOFA-O.

Se realizó el cálculo de tamaño de la muestra para la frecuencia en una población en el programa OpenEpi. Se tuvo en cuenta una prevalencia para el ingreso a $\mathrm{UCl}$ de pacientes con sepsis de $15 \%$ en países en vías de desarrollo, con una confianza de $95 \%$ para un total de 51 pacientes.

Se realizó un censo de las pacientes que cumplieron los criterios de inclusión para sepsis obstétrica, llevándose a cabo mediante la escala SOS y SOFA-O a su ingreso a la UCI mediante signos vitales y parámetros de laboratorio. Se enumeraron los factores de riesgo para el desarrollo de sepsis, los cuales se dividieron en factores prenatales (infección de vías urinarias, anemia/malnutrición/desnutrición, DM materna, bajo nivel socioeconómico, pobre control prenatal, obesidad y vaginosis bacteriana diagnosticada en el embarazo), factores relacionados con parto y puerperio (hemorragia intraparto mayor de 500 mL o en cesárea mayor de 1,000 mL, corioamnioitis en embarazo a término, o pretérmino, trabajo de parto prolongado, alumbramiento incompleto/ retención de restos placentarios y ruptura de membranas mayor de seis horas) y procesos invasivos. Se determinaron las variables de tratamiento en la Unidad de Cuidados Intensivos.

Se analizaron las variables de las escalas SOS y SOFA-O y se correlacionaron con mortalidad cada una de ellas con valor de p e intervalo de confianza.

Se determinaron las fallas orgánicas, así como los resultados finales en las pacientes, para exponer el porcentaje de cada una de ellas. Se calculó el porcentaje de mortalidad.

Análisis estadístico: Se realizó correlación de variables y se analizaron. Se mostró relación con dos variables mediante $\mathrm{p}$ de Pearson para las cuantitativas y Spearman para las cualitativas, además de t de Student para las variables cuantitativas de distribución normal y U de Mann-Whitney para las de libre distribución. La información obtenida se manejó en una base de datos 
y se analizó a través de estadística descriptiva y analítica mediante el programa SPSS versión 23 , además de regresión de Cox para supervivencia.

Criterios de inclusión: pacientes obstétricas de cualquier edad con diagnóstico de sepsis que ingresaron a UCI. Pacientes obstétricas de cualquier edad gestacional o en el puerperio que tengan datos de sepsis en UCl. Pacientes con cualquier diagnóstico de patología obstétrica que desarrollaron sepsis durante su estancia en UCI. Pacientes obstétricas que hayan fallecido durante la estancia en $\mathrm{UCl}$ con diagnóstico de sepsis obstétrica.

Criterio de no inclusión: pacientes que no cumplan criterios de sepsis. Pacientes en cuya historia clínica no estén consignados los parámetros correspondientes a las variables evaluadas en las escalas SOS y SOFA-O para realizar el cálculo de puntaje. Pacientes con diagnóstico de embarazo molar.

Criterios de eliminación: pacientes con diagnóstico de sepsis, pero que hayan sido trasladadas a otra unidad.

\section{Consideraciones éticas aplicables al estudio}

Este estudio se ajusta a las Normas Éticas Institucionales y a la Ley General de Salud en materia de investigación, así como a la Declaración de Helsinki en la décima octava Asamblea Mundial en 1964, ratificada y perfeccionada en el Congreso de Tokio, Japón, en 1975; en Venecia, en 1983; en Hong Kong, en 1989; en Sudáfrica, en 1996, y en la 52ª Asamblea General en Edimburgo. También se ajusta a las normas y procedimientos en materia de investigación que rigen en las instituciones de salud.

La investigación se inicia una vez que sea aprobada por el Comité Local de Investigación.

De acuerdo con el reglamento de la ley General de Salud en materia de investigación para la salud en su artículo 17, se considera un estudio sin riesgo.

\section{RESULTADOS}

Se realizó un estudio clínico, prospectivo y longitudinal, el cual incluyó una población total de 284 pacientes obstétricas que ingresaron a $\mathrm{UCI}$, de las cuales 51 se seleccionaron de entre quienes tuvieron todos los criterios de inclusión para sepsis obstétricas, las cuales se determinaron mediante la escala SOS y SOFA-O.

En cuanto a edad, las pacientes con sepsis obstétrica tuvieron una media de 24 años con un rango intercuartilar (18-30 años). De las 51 pacientes, 24 eran primigestas con un porcentaje de $47 \%$ y multigestas 27 pacientes, con un porcentaje de $52.9 \%$.

Del total de las pacientes se dividieron factores de riesgo y causas maternas y obstétricas; de los factores de riesgo materno los principales son la anemia y la desnutrición, con presencia en 30 pacientes (70.6\%), seguidas de sobrepeso en $15(29.4 \%)$, diabetes tipo 2 en $12(23.5 \%)$ y sobrepeso en $12(23.5 \%)$. De los factores y causas obstétricas fueron parto por cesárea en 50 pacientes (98\%), pobre control prenatal en $26(51 \%)$, un trabajo de parto prolongado en ocho (15.7\%), ruptura prematura de membranas en seis pacientes $(11.8 \%)$, infección de vías urinarias en cinco (9.8\%), deciduitis en dos pacientes (3.9\%) y vaginosis bacteriana en el último trimestre en dos (3.9\%).

Los procedimientos invasivos que tuvieron las pacientes fueron manejo de vía aérea avanzada en 15 de las 51 pacientes, por causas perioperatorias, que corresponden a $29.4 \%$ del total; en las 51 pacientes (100\%) con diagnóstico de sepsis se colocó una vía venosa central con canalización de subclavia (Tabla 1).

Durante su estancia en la Unidad de Cuidados Intensivos se continuó reanimación de estas pacientes. Los hemoderivados que se requirieron fueron concentrados eritrocitarios en número de $31(60.7 \%)$, plasma fresco congelado, 22 unidades (43.1\%) y 16 aféresis plaquetarias $(31.3 \%)$. Se utilizaron cristaloides en todas las pacientes (100\%); el balance hídrico total hasta su

Tabla 1: Características generales de las pacientes con sepsis.

\begin{tabular}{lc}
\hline Variables & $\mathrm{n}=51$ \\
$\mathrm{n}(\%)$ \\
\hline Edad (RIC) & $24(18-30)$ \\
Gesta & \\
$\quad$ Primigesta & $24(47.0)$ \\
Multigesta & $27(52.9)$ \\
Diagnóstico & \\
Choque hipovolémico/atonía uterina & $10(19.6)$ \\
Choque hipovolémico/emb. ectópico roto & $1(1.9)$ \\
Eclampsia & $2(3.9)$ \\
HELLP incompleto & $2(3.9)$ \\
Hemorragia obstétrica & $1(1.9)$ \\
Preeclampsia severa & $27(52.9)$ \\
Puerperio patológico postcesárea & $1(1.9)$ \\
Síndrome de HELLP & $7(13.7)$ \\
Factores de riesgo materno & \\
Diabetes tipo 2 & $12(23.5)$ \\
Bajo nivel socieconómico & $12(23.5)$ \\
Sobrepeso & $15(29.4)$ \\
Anemia/malnutrición & $30(70.6)$ \\
Factores de riesgo obstétrico & \\
Control prenatal & $26(51.0)$ \\
Ruptura prematura de membranas & $6(11.8)$ \\
Trabajo de parto prolongado & $8(15.7)$ \\
Infección de vías urinarias & $5(9.8)$ \\
Vaginosis bacteriana & $2(3.9)$ \\
Deciduitis & $2(3.9)$ \\
Corioamnioitis & $6(11.8)$ \\
Parto por cesárea & $50(98.0)$ \\
Procedimientos invasivos & \\
Colocación de catéter venoso central & $51(100.0)$ \\
Manejo de vía aérea avanzada & $15(29.4)$ \\
\hline
\end{tabular}


egreso fue de $543 \mathrm{~mL} \pm 528 \mathrm{~mL}(-513 \mathrm{~mL}$ a $1,599 \mathrm{~mL})$. Se requirió de tratamiento de sustitución renal en 13 pacientes $(7.7 \%)$ mediante hemodiálisis intermitente. Se utilizaron vasopresores en 10 pacientes (19.6\%) y la dosis fue desde $0.01 \mu \mathrm{g} / \mathrm{kg} / \mathrm{min}$ hasta $1 \mu \mathrm{g} / \mathrm{kg} / \mathrm{min}$; de las 15 pacientes que requirieron manejo de vía aérea avanzada cinco pacientes $(9.8 \%)$ sólo requirieron un día de ventilación mecánica, seis (12.8\%) dos días, dos pacientes $(3.9 \%)$ con cinco días y otras dos $(3.9 \%)$ nueve días de ventilación mecánica. Se realizaron cultivos de herida quirúrgica, hemocultivos, y para pacientes con intubación orotraqueal se realizaron cultivos de secreción bronquial, con crecimiento de Staphylococcus aureus en herida quirúrgica en tres pacientes (5.8\%), crecimiento de Klebsiella pneumoniae en secreción bronquial en una paciente $(1.9 \%)$, crecimiento de E. coli $\mathrm{BLEE}+$ en herida quirúrgica en cuatro pacientes y dos en secreción bronquial, en un total de seis pacientes $(11.7 \%)$ y crecimiento de estafilococo epidermidis en hemocultivo central en una paciente (1.9\%). En cuanto al uso de antibióticos, se prescribieron a todas las pacientes; los grupos de éstos fueron: cefalosporinas en ocho pacientes (16\%), uso de carbapenémico en $12(23.5 \%)$ cefalosporina + lincosamidas en 15 (29\%), carbapenémico + nitroimidazol en $11(21.5 \%)$, cefalosporina + nitroimidazol en $11(21.5 \%)$ y glucopéptido + carbapenémico en tres (6\%). Sólo a cinco pacientes (6\%) se indicó antimicótico triazol (Tabla 2).

Para el diagnóstico y pronóstico en pacientes con sepsis obstétrica se realizó el instrumento de escalas SOS y SOFA-O; el puntaje para todas fue una mediana de 7 puntos con un rango intercuartilar (6-8) y una mediana de 4 con un rango intercuartilar (4-5).

Las fallas orgánicas principales fueron: hematológica en 16 pacientes (33.1\%), seguida de falla renal en 14 pacientes $(27.4 \%)$ y cardiovascular en 10 pacientes (19.6\%); las menores fallas fueron la hepática y la neurológica (Tabla 2).

Se correlacionaron las variables de cada escala SOS y SOFA-O para mortalidad materna (Figura 1). Las variables calculadas fueron: plaquetas para muerte materna con una mediana de 42,500 con un RIC $(34,000-$ $296,250)$ con una $p=0.509$ con IC 95\% (156.6-723.7). La creatinina tuvo una media de $3.25 \pm 1.1 \mathrm{mg} / \mathrm{dL}$ con una $p=0.02$ con un IC $95 \%$ (1.11-3.05); bilirrubinas totales, una media de 2.8 con una DE 0.85 , una $p=0.01$ y un IC de $95 \%$ (1.6-2.4), la relación $\mathrm{PaO}_{2} / \mathrm{FIO}_{2}$ con una mediana de 99 con un RIC de 82.5-125 con una p 0.015 con un IC 95\% (86.3-202). En cuanto a balance hídrico total tuvieron una media de $1,385 \pm 1,410 \mathrm{~mL}$ con una $p$ $=0.036$ con un IC 95\% (1,326-3,153), presentaron una saturación de $\mathrm{O}_{2}$ arterial una mediana de $85 \%$ con un RIC (84-89) con una $p=0.218$ con un IC 95\% (80-94), la frecuencia respiratoria con una mediana 27 con un RIC ( 25-28) con una $p=0.26$, la PAS con una media de $77 \pm 11.9 \mathrm{mmHg}$ con una $p=0.116$ con un IC $95 \%$
(23.9-25.7); en frecuencia cardiaca se obtuvo una mediana de 137 con un RIC (135-143) y una $p=0.008$ con un IC 95\% (17.13-30.96); la temperatura presentó una mediana de $35.4{ }^{\circ} \mathrm{C}$ con un mínimo de 34.9 y un máximo de 39 con una $p=0.314$ y un IC 95\% (3.26-3.84); la hemoglobina tuvo una mediana de $7.75 \mathrm{~g} / \mathrm{dL}$ con un RIC (0.97-12.5) con una $p=0.405$ con un IC $95 \%$ (0.75-5.07); el lactato una media de $5.5 \pm 1.2 \mathrm{mmol}$ con una $p=0.011$ con un IC 95\% (1.49-5.39); la saturación venosa central tuvo una mediana de $53 \%$ con un RIC (47-57) con una $p=0.001$ y un IC $95 \%$ (15.4-33), los leucocitos tuvieron una mediana de $15.1 \times 10^{3}$ con un RIC (16.8-35) con una $p=0.21$ y un IC 95\% (5.77-18.7); la dosis de vasopresores con una mediana de $0.55 \mu \mathrm{g} /$

\section{Tabla 2: Tabla de intervenciones y desenlace en la Unidad de Cuidados Intensivos en pacientes con sepsis obstétrica}

\begin{tabular}{lcl}
\hline Días de ventilación mecánica & \\
Ninguno & $36(70.6)$ \\
1 & $5(9.8)$ \\
2 & $6(11.8)$ \\
5 & $2(3.9)$ \\
9 & $2(3.9)$ \\
Uso de antibióticos & $51(100.0)$ \\
Grupos de antibióticos & \\
Carbapenémico & $12(23.5)$ \\
Cefalosporinas & $8(16.0)$ \\
Cefalosporinas + lincosamidas & $15(29.0)$ \\
Cefalosporinas + nitroimidazol & $2(4.0)$ \\
Carbapenémico + nitroimidazol & $11(21.5)$ \\
Glucopéptido + carbapenémico & $3(6.0)$ \\
Antifúnfico (tinidazol) & $5(5.8)$ \\
Realización de cultivos & $51(100.0)$ \\
Microorganismos aislados & \\
S. aureus & $3(5.8)$ \\
E. coli BLEE+ & $6(11.7)$ \\
S. epidermidis & $1(1.9)$ \\
Klebsiella pneumoniae & $1(1.9)$ \\
Uso de vasopresores & $10(19.6)$ \\
Dosis de vasopresores $\mu g /$ /kg/min (mín.-máx.) & $0.01(0.01-1)$ \\
Días de vasopresores (mín.-máx.) & $7(1-9)$ \\
Balance hídrico total mL (DE) & $543.14( \pm 528.5)$ \\
Cristaloides & $51(100)$ \\
Hemoderivados & $34(66.6)$ \\
Tipo de hemoderivados & \\
Concentrado eritrocitario & $31(60.7)$ \\
Plasma fresco congelado & $22(43.1)$ \\
Plaquetas & $16(31.3)$ \\
Uso de hemodiálisis intermitente & $13(7.7)$ \\
Días de estancia en UCl (mín.-máx.) & $4(2-17)$ \\
Fallas orgánicas & \\
Falla neurológica & $4(7.8)$ \\
Falla respiratoria & $3(5.8)$ \\
Falla hepática & $4(7.8)$ \\
Falla cardiovascular & $10(19.6)$ \\
Falla renal & $14(27.4)$ \\
Falla hematológica & $16(31.3)$ \\
Puntaje de escalas & $7(6-8)$ \\
SOS (RIC) & $4(4-5)$ \\
Mortalidad & & \\
& & \\
& & \\
& & \\
&
\end{tabular}



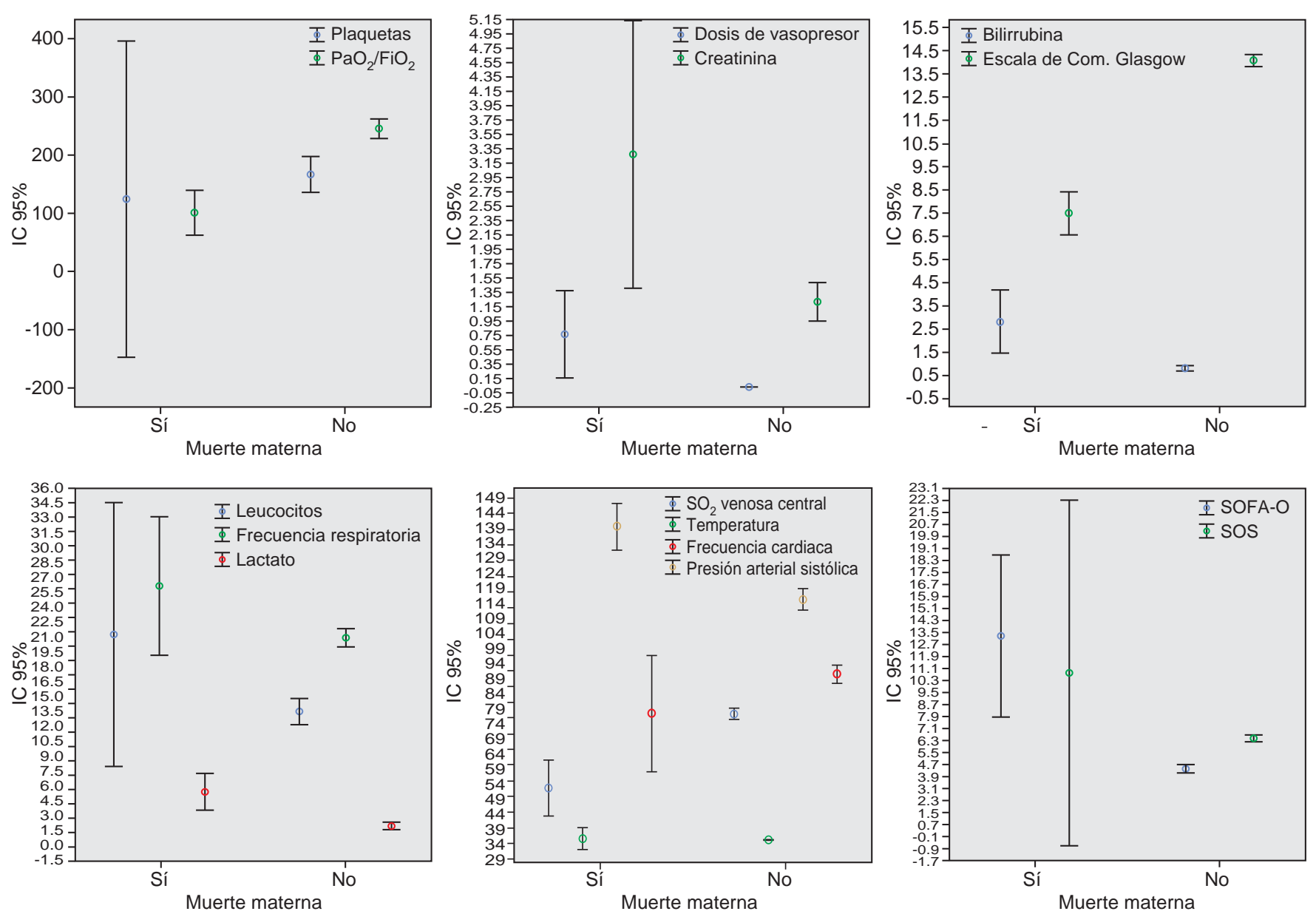

Figura 1: Correlación de variables y escalas con mortalidad materna.

$\mathrm{kg} / \mathrm{min}$ con un mínimo de 0.11 y un máximo de $1.34 \mu \mathrm{g} /$ $\mathrm{kg} / \mathrm{min}$ (Tabla 3).

Se calculó el porcentaje de muerte materna siendo en cuatro pacientes de $7.8 \%$ para esta población. Se realizó regresión Cox para SOFA obstétrico y supervivencia acumulada encontrando que a partir de 4 puntos disminuye la supervivencia hasta en $50 \%$, mientras que para el SOS la supervivencia disminuye hasta en $60 \%$ a partir de 6 puntos (Figura 2).

\section{DISCUSIÓN}

La sepsis representa un alto índice de morbimortalidad; es la principal causa de muerte materna con un porcentaje de $15 \%$ en todo el mundo ${ }^{1}$; para nuestro estudio fue de $7.8 \%$.

Las causas de sepsis materna incluyen cesárea, episiotomía, arrastre de bacterias del tracto genital, ruptura prolongada de membranas y múltiples revisiones vaginales. ${ }^{2}$ Como en nuestro estudio, las causas obstétricas fueron la cesárea, el trabajo de parto prolongado, junto con un factor de riesgo principal como lo es el control prenatal, mientras entre las causas y factores maternos se encuentra la anemia. Complicaciones maternas como la preeclampsia, la hemorragia y la sepsis constituyen las causas obstétricas más comunes de ingreso a la $\mathrm{UCl} .{ }^{9} \mathrm{El}$ diagnóstico inicial principal de las pacientes con sepsis obstétricas fue preeclampsia severa en 27 pacientes quienes representaron $52.9 \%$ de toda la población, seguida de choque hipovolémico en 10 pacientes correspondiendo a $19.6 \%$. Los resultados coincidieron con lo reportado en la literatura a nivel mundial.

Cuando se pierde el equilibrio entre el aporte y la utilización de oxígeno por parte de las células, las alteraciones metabólicas que se suscitan a nivel intracelular pueden ocasionar la muerte celular y el desarrollo de falla orgánica, incluso la muerte del paciente. Las pacientes obstétricas constituyen una población única en virtud de los diversos cambios anatómicos, bioquímicos y fisiológicos que se producen durante el estado de gravidez y el puerperio. ${ }^{4}$ En nuestro estudio se relacionaron las variables fisiológicas y bioquímicas. En la escala SOS y SOFA-O encontramos mayor co- 
Tabla 3: Correlación de variables fisiológicas, bioquímicas escala SOS y SOFA-O con muerte materna.

\begin{tabular}{|c|c|c|c|}
\hline Variables & $\begin{array}{l}\text { Muerte materna } \\
\qquad \mathrm{n}=4\end{array}$ & $\begin{array}{l}\text { Sobrevivientes } \\
\quad n=47\end{array}$ & $\mathrm{p}$ \\
\hline Plaquetas mediana (RIC) & $\begin{array}{c}42500 \\
(34.000-296.250)\end{array}$ & $\begin{array}{c}177,000 \\
(48750-245.000)\end{array}$ & 0.509 \\
\hline $\begin{array}{l}\text { Leucocitos mediana (RIC) } \\
10 x^{3}\end{array}$ & $15.1(16.8-35)$ & $8.2(3.5-8.9)$ & 0.210 \\
\hline Creatinina media (DE) mg/dL & $3.25(1.1)$ & $0.92(0.3)$ & 0.020 \\
\hline $\mathrm{BT}(\mathrm{DE}) \mathrm{mg} / \mathrm{dL}$ & $2.8(0.85)$ & $0.82(0.33)$ & 0.010 \\
\hline $\mathrm{PaO}_{2} / \mathrm{FiO}_{2}$ mediana $(\mathrm{RIC})$ & $99(82.5-125000)$ & $249(174.1-287.5)$ & 0.010 \\
\hline $\begin{array}{l}\text { Balance hídrico total media } \\
\text { (DE) mL }\end{array}$ & $+1385( \pm 1410)$ & $+469( \pm 310)$ & 0.036 \\
\hline $\mathrm{SO}_{2} \%$ mediana $(\mathrm{RIC})$ & $86(84-89)$ & $92(89-95)$ & 0.218 \\
\hline FR mediana $(\mathrm{RIC})$ & $27(25-28)$ & $22(20-25)$ & 0.260 \\
\hline PAS mmHg media DE & 77 (11.9) & $89(9.7)$ & 0.116 \\
\hline FC mediana (RIC) & $137(135-143)$ & $115(105-120)$ & 0.008 \\
\hline $\begin{array}{l}\text { Temperatura mediana } \\
\text { (máx.-mín.) }\end{array}$ & $35.4(34.9-39)$ & $34(35-37)$ & 0.314 \\
\hline $\begin{array}{l}\text { Hemoglobina (mediana) (RIC) } \\
\mathrm{g} / \mathrm{dL}\end{array}$ & $7.75(3.97-12.5)$ & $10.7(9-11.9)$ & 0.405 \\
\hline Lactato media (DE) mmol & $5.5(1.2)$ & $2.08(0.6)$ & 0.011 \\
\hline $\mathrm{SO}_{2} \%$ venosa(mediana) (RIC) & $53(47-57)$ & $76(72-80)$ & 0.001 \\
\hline $\begin{array}{l}\text { Dosis de vasopresores } \\
\text { Mediana (mín.-máx.) } \\
\mu \mathrm{g} / \mathrm{kg} / \mathrm{min}\end{array}$ & $0.55(0.11-1.34)$ & $0.014(0.01-0.016)$ & 0.005 \\
\hline Glasgow mediana (mín.-máx.) & $8(7-8)$ & $13(12-15)$ & 0.001 \\
\hline SOS mediana (mín.-máx.) & $7(7-22)$ & $6(6-8)$ & 0.030 \\
\hline SOFA-O mediana (mín.-máx.) & $13(10-18)$ & $4(3-7)$ & 0.001 \\
\hline
\end{tabular}

rrelación y significancia en las variables de creatinina sérica con una $p>0.01$, encontrándose que de las 14 pacientes que hicieron falla renal 13 de ellas tuvieron tratamiento sustitutivo renal con $\mathrm{HDI}$, siendo la segunda falla orgánica frecuente en pacientes sépticas, la $\mathrm{PaO}_{2} / \mathrm{FiO}_{2}$ con una $\mathrm{p} 0.001$, con una adecuada correlación para mortalidad, a pesar de que la falla respiratoria fue infrecuente para esta población. Las pacientes con falla cardiovascular se evaluaron mediante la PAS y mediante el uso de vasopresores, teniendo en cuenta que la PAS no tiene una adecuada significancia ya que presenta una $p$ de 0.116 , mientras la frecuencia cardiaca tuvo mejor correlación y significancia con una p 0.008 y un IC 95\% (17.13-30.96). El lactato es una medida para valorar el estado de choque, y lo encontramos en nuestro estudio con una $p 0.01$ y un IC 95\% (1.49-5.39).

La falla hematológica fue la principal falla orgánica en nuestro grupo; sin embargo, al correlacionarse con la mortalidad las plaquetas no tienen una adecuada correlación ni significancia presentando una p de 0.509.

El tratamiento es la piedra angular de toda paciente obstétrica; la reanimación en las pacientes con sepsis debe estar encaminada a la pronta consecución de valores fisiológicos adecuados y continuar el proceso de reanimación mediante volumen de calidad, como son los hemoderivados. ${ }^{9}$ Los hemoderivados que más se utilizaron fueron concentrados eritrocitarios en $60.7 \%$,
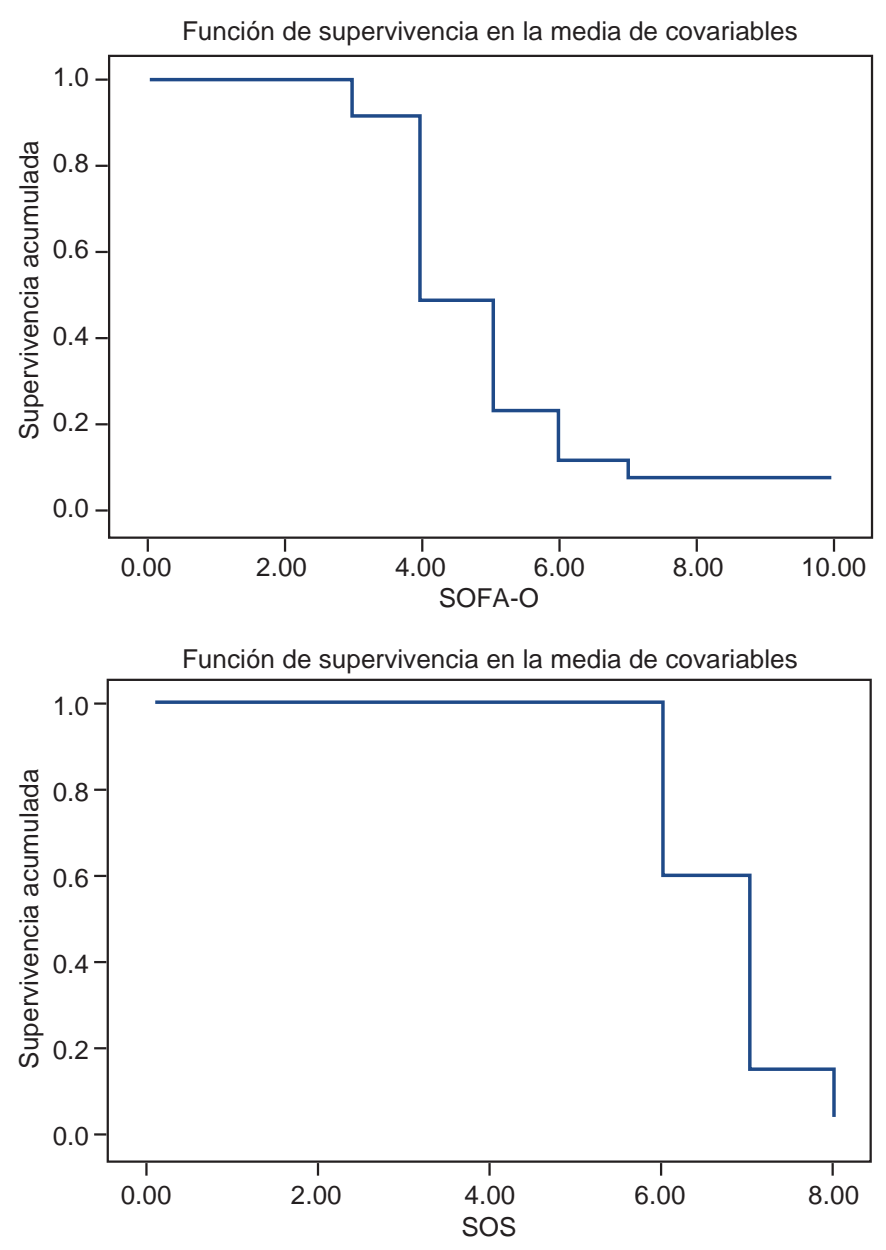

Figura 2: Regresión de Cox para supervivencia en SOFA-O y en SOS.

seguidos del plasma fresco congelado con $43.1 \%$. Por último, aféresis plaquetaria en $31.3 \%$. No se utilizaron crioprecipitados, ya que el valor de fibrinógeno no se tuvo en todos los casos, por lo cual se excluyó esta variable.

Los balances de líquidos que llegan a tener las pacientes son predictores de mortalidad, ya que estas pacientes deberán mantener volemia adecuada según necesidad y guías de práctica clínica o protocolos del servicio; sin embargo, en el puerperio la posibilidad de edema pulmonar por sobrecarga de volumen es mayor. ${ }^{9,13-15}$ El balance negativo mayor fue de $1,435 \mathrm{~mL}$ y el mayor positivo $4,205 \mathrm{~mL}$, el cual se comenta que entre mayor sobrecarga, mayor falla renal y mayor mortalidad.

Los organismos que causan infección son por lo general de la flora polimicrobiana del tracto genitourinario. ${ }^{10-12}$ Los organismos que se aislaron en estas pacientes con cultivos positivos cuantitativos fueron en primer lugar E. coli BLEE+ en $11.7 \%$, seguida de Staphylococcus aureus en 5.8\% y Klebsiella pneumoniae en $1.9 \%$ de los casos. Los demás cultivos sin desarrollo, 
por lo que los cultivos negativos no sirven para excluir pacientes con sospecha de sepsis. Los esquemas antibióticos intravenosos combinados que se recomiendan son: ceftriaxona + clindamicina + ampicilina. Betalactámicos + aminoglucósidos. Clindamicina + aminoglucósidos o quinolonas. Betalactámicos + aminoglucósidos + metronidazol. Cefalosporinas de tercera generación + quinolona. Carbapenémicos + vancomicina. ${ }^{14}$ Como en nuestro estudio se utilizaron mayormente la combinación de cefalosporinas (ceftriaxona) + lincosamidas (clindamicina) en $29.4 \%$ de los casos.

En las pacientes con sepsis obstétrica se diagnosticó mediante escalas; una de éstas es la escala SOS, que es un sistema de puntaje modificado que identifica el riesgo de ingreso a la Unidad de Cuidados Intensivos (UCI) de pacientes obstétricas con sepsis. El puntaje SOS es una escala validada, utilizada en estudios previos donde ha demostrado un adecuado rendimiento diagnóstico, con un valor corte de $\geq 6$ puntos. ${ }^{11}$ En nuestro estudio esta escala presentó una mediana de 7 con un mínimo de 7 y máximo de 22 puntos con relación a muerte materna con una p 0.030 , mientras que la escala SOFA-O, es una modificación de la escala utilizada para cualquier paciente, sólo con diferencia en variables fisiológicas maternas como es en lo respiratorio, y si presentan o no sepsis; dicha escala nos apoya para predecir fallas orgánicas y mortalidad. , $^{3,12}$ En nuestro estudio, la mediana del puntaje fue de 13 puntos con un mínimo de 10 y máximo de 18 puntos en relación a muerte materna con una $p$ de 0.001 .

\section{CONCLUSIONES}

La paciente con sepsis obstétrica crítica representa un reto para los intensivistas, desde la sospecha clínica con variables fisiológicas hasta el diagnóstico con escalas validadas; sin embargo, cada una de ellas requiere de variables fisiológicas y bioquímicas que reclaman mayor tecnología, por lo que se deberán validar parámetros fáciles de emplear que no requieran de mayor tecnología, como lo es un «cheklist» para pacientes que no se encuentren en áreas críticas, y el diagnóstico pueda ser sospechado por cualquier personal de salud sin tener retraso en el mismo y en la terapia.

Con una adecuada reanimación individualizada se evitarán complicaciones mayores. El reconocimiento rápido de la infección y la terapia adecuada impactarán en la supervivencia de la paciente.

\section{BIBLIOGRAFÍA}

1. Acosta C, Knight M. Sepsis and maternal mortality. Curr Opin Obstet Gynecol. 2013;25(2):109-116.

2. Lucas D, Robinson P, Nel M. Sepsis in obstetrics and the role of the anaesthetist. Int J Obst Anesth. 2012;21:56-67.

3. SINAVE/DGE/Salud/Sistema de Notificación Inmediata de MM 2016 y 2017.

4. Morgan J, Roberts S. Maternal sepsis. Obstet Gynecol Clin North Am. 2013;400:69-87.

5. Chebbo A, Tan S, Kassis C, Tamura L, Carlson RW. Maternal sepsis and septic shock. Crit Care Clin. 2016;32(1):119-135.

6. Bauer ME, Bauer ST, Rajala B, MacEachern MP, Polley LS, Childers D, et al. Maternal physiologic parameters in relationship to systemic inflammatory response syndrome criteria: a systematic review and meta-analysis. Obstet Gynecol. 2014;124(3):535-541.

7. Barton J, Sibai B. Severe sepsis and septic shock in pregnancy. Obstet Gynecol. 2012;120:689-706.

8. Pacheco L, Saade G. Severe sepsis during clin. Obstet Gynecol. 2014;57(4):827-834.

9. Fernández-Pérez ER, Salman S, Pendem S, Farmer JC. Sepsis during pregnancy. Crit Care Med. 2005;33:S286-293.

10. Albright CM, Ali TN, Lopes V, Rouse DJ, Anderson BL. The sepsis in obstetrics score: a model to identify risk of morbidity from sepsis in pregnancy. Am J Obstet Gynecol. 2014;211(1):39. e1-8.

11. Aarvold $A B$, Ryan HM, Magee LA, von Dadelszen $P$, Fjell $C$, Walley KR. Multiple organ dysfunction score is superior to the obstetric-specific sepsis in obstetrics score in predicting mortality in septic obstetric patients. Crit Care Med. 2017;45(1):e49-e57.

12. Jain S, Guleria K, Suneja A, Vaid NB, Ahuja S. Use of the Sequential Organ Failure Assessment score for evaluating outcome among obstetric patients admitted to the intensive care unit. Int J Gynaecol Obstet. 2016;132(3):332-336.

13. Rimawi B, Soper D, Eschenbach D. Group A streptococcal infections in obstetrics and gynecology. Clin Obstet Gynecol. 2012;50:864-874.

14. Olvera L, Dutra D. Early recognition and management of maternal sepsis. Nurs Womens Health. 2016;20;2:182-189.

15. Pollock W, Rose L, Dennis CL. Pregnant and postpartum admissions to the intensive care: a systemic review. Intensive Care Med. 2010;36:1465-1474.

Conflicto de intereses: Este trabajo no tiene conflicto de intereses.

Correspondencia:

Dra. Blanca Estela Herrera Morales

Monumento a La Raza Núm. 12,

Col. Metropolitana 2da Sección,

Ciudad Nezahualcóyotl, 57740, Estado de México.

Tel: 22325448

E-mail: blanquis_k9@hotmail.com 